\title{
Falla cardíaca como manifestación temprana de hipertiroidismo neonatal. Caso clínico
}

\author{
JORGE LUIS ALVARADO S. ${ }^{1}$, ZHIRLY ANDREA FERNÁNDEZ V. ${ }^{2}$, \\ 1. Pediatra neonatólogo. Fundación Cardiovascular de Colombia, Floridablanca, Santander. Departamento de Pediatría. \\ Unidad de Neonatología. \\ 2. Pediatra. Salud Total, Empresa Promotora de Salud. Consulta externa.
}

\begin{abstract}
Heart failure as early manifestation of neonatal hyperthyroidism. Case report

Neonatal hyperthyroidism is usually a self-limited condition frequently associated with transplacental passage of thyroid stimulating antibodies secondary to maternal autoimmune disorders. To timely detect mothers with this medical antecedents decreases the risk for fetal adverse events. Objective: To report a case of neonatal hyperthyroidism associated with intrauterine growth restriction and heart failure. Case report: A 36 week-old newborn with birth weight of 1,240 g. Symptoms were tachycardia, distal coldness, exophthalmos, hepatomegaly and tremors. Echocardiogram ruled out structural heart disorders. Due to maternal symptoms suggestive of hyperthyroidism, TSH tests were performed showing $0.01 \mathrm{ulU} / \mathrm{ml}$, free T4 $7.7 \mathrm{ng} / \mathrm{dl}$, so the diagnosis of neonatal hyperthyroidism was confirmed. It was treated with methimazole and propanol, alleviating the symptoms and decreasing the levels of free T4. Conclusions: To know the maternal history helps identify and manage neonatal complications of hyperthyroidism. Heart failure and other cardiopulmonary disorders are determinants of mortality during early neonatal period. High-risk newborns should receive follow up assessments. (Key words: Hyperthyroidism, Graves' disease, neonatal, cardiac failure).
\end{abstract}

Rev Chil Pediatr 2014; 85 (2): 207-212

\section{RESUMEN}

Introducción: Hipertiroidismo neonatal es una condición usualmente autolimitada, generalmente asociada al paso transplacentario de anticuerpos estimulantes de tiroides, secundario a enfermedades autoinmunes maternas. Detectar oportunamente a las madres con estos antecedentes, permitirá disminuir el riesgo de eventos adversos fetales. Objetivo: Presentar un caso de hipertiroidismo neonatal, asociado a falla cardíaca y restricción del crecimiento intrauterino, Caso clínico: Recién nacido de 36 semanas, peso de nacimiento $1.240 \mathrm{~g}$. Evolucionó con taquicardia, frialdad distal, exoftalmos, hepatomegalia y temblores. Ecocardiograma descartó alteración cardíaca estructural. Por hallazgos maternos sugerentes de hipertiroidismo, se realizaron exámenes encontrando TSH de 0,01 uUI/ml, T4 libre de 7,7 ng/dl, con lo que se confirmó el diagnóstico de hiperti-

Recibido el 21 de junio de 2013, devuelto para corregir el 2 de agosto de 2013, segunda versión 15 de septiembre de 2013 , aceptado para publicación el 28 de enero de 2014.

Este trabajo cumple con los requisitos sobre consentimiento /asentimiento informado, comité de ética, financiamiento, estudios animales y sobre la ausencia de conflictos de intereses según corresponda.

Correspondencia a:

Jorge Luis Alvarado Socarras

E-mail: Jorgealso2@yahoo.com 
roidismo neonatal. Se manejó con metimazol y propanolol con resolución de los síntomas y descenso de los niveles de T4 libre. Conclusiones: Conocer los antecedentes maternos permite identificar y manejar las complicaciones neonatales del hipertiroidismo. La falla cardíaca y otras alteraciones cardiopulmonares son los factores determinantes de la mortalidad en el período neonatal temprano. Debe haber un seguimiento a los recién nacidos de riesgo.

(Palabras clave: Hipertiroidismo, enfermedad de Graves, neonatal, falla cardíaca).

Rev Chil Pediatr 2014; 85 (2): 207-212

\section{Introducción}

La falla cardíaca en el período neonatal puede ser secundaria a enfermedades congénitas o adquiridas, de etiología múltiple, tanto estructurales, metabólicas o ambientales. E1 diagnóstico diferencial va a depender del momento de presentación. Hay síntomas precoces (primeras horas de vida) con cuadros de asfixia, alteraciones metabólicas y sepsis. Durante la primera semana aparecen las cardiopatías congénitas, principalmente las ductus dependientes, falla cardíaca secundaria a la disminución de la reserva miocárdica por prematurez, y alteraciones endocrinas como la insuficiencia adrenal y el hipertiroidismo ${ }^{1,2}$.

La falla cardíaca es una manifestación temprana del hipertiroidismo neonatal, el cual es una condición muy rara, con una incidencia de 1: 50.000 nacidos vivos, asociado a una mortalidad de aproximadamente el $25 \%$, debida a alteraciones cardiopulmonares u otras complicaciones como infecciones y trombocitopenia. Además se asocia a retraso del neurodesarrollo, craneosinostosis e hipertensión pulmonar persistente $^{3}$. Otras manifestación clínicas frecuentes son restricción del crecimiento intrauterino, bocio, irritabilidad, temblores, exoftalmos, edema periorbitario, signos de hipermetabolismo (pérdida de peso, diarrea, sudoración), acrocianosis, hepatoesplenomegalia y petequias ${ }^{4}$.

El hipertiroidismo neonatal se desarrolla entre el $1-2 \%$ de los recién nacidos (RN) de embarazadas que presenta enfermedad de Graves, y es secundario al paso transplacentario de anticuerpos estimulantes del tiroides (TRSab) al unirse al receptor de tirotropina fetal, incrementando la función tiroidea o por mutaciones activas del receptor de tirotropina y subunidad alfa de la proteína $\mathrm{G}^{3,5}$.
La enfermedad de Graves se presenta en el $0,2 \%$ de todas las gestaciones, y si no es tratada de forma adecuada, puede presentar complicaciones tales como parto de pretérmino, aborto, preeclampsia, trombosis, tormenta tiroidea y abruptio placentario, por lo que el diagnóstico precoz es relevante ${ }^{6}$.

Adicional a lo anterior, existen riesgos fetales y neonatales. En estos últimos, puede haber síntomas precoces o tardíos, lo que amerita un seguimiento durante las primeras semanas con examen clínico y de laboratorio. El pronóstico temprano está determinado por el compromiso cardíaco, sin embargo, a largo plazo puede haber compromiso neurológico incluyendo retardo mental ${ }^{7}$.

El objetivo es presentar un caso de hipertiroidismo neonatal asociado a falla cardíaca $\mathrm{y}$ restricción del crecimiento intrauterino, $\mathrm{La}$ orientación diagnóstica fue hecha en base al fenotipo materno (enfermedad de Graves). Contrario a lo usual, el diagnóstico y manejo materno fue realizado después de pesquisar la morbilidad neonatal.

\section{Caso clínico}

Recién nacido hijo de madre primigesta de 18 años, con embarazo de 36 semanas, con control prenatal irregular, quien presentó parto vaginal y líquido amniótico con meconio. Pesó al nacer $1.240 \mathrm{~g}(<\mathrm{p} \mathrm{3})$, midió $41 \mathrm{~cm}(<\mathrm{p} 3)$, con apgar de 7 y 9 . Presentó dificultad respiratoria temprana que requirió soporte ventilatorio y surfactante pulmonar, con adecuada respuesta. Se logró extubación a CPAP, pero presentó taquicardia y taquipnea persistente, por lo cual se realizó ecocardiograma sugestivo de coartación de aorta por lo que fue deri- 
vado a un centro de mayor complejidad, a los 13 días de vida.

A su ingreso a la institución se tomó presión arterial en cuatro extremidades: Miembros superiores derecho e izquierdo 88/49 presión arterial media (PAM) 62 y 90/52 PAM 63, miembros inferiores derecho e izquierdo 86/43 PAM 61, 78/44 PAM 53, frecuencia cardíaca 180 y respiratoria $65 \mathrm{x}$ min. Al examen segmentario destacaba hepatomegalia, frialdad distal, taquicardia, soplo en foco pulmonar II/ VI, pulsos femorales presentes, retracción subcostal, temblor distal fino, exoftalmo marcado y precordio hiperdinámico (figura 1).

Se realizó Ecocardiograma que descartó coartación aórtica, pero mostró disfunción miocárdica que requirió soporte inotrópico. Ecografía transfontanelar y abdominal normales. En los exámenes de laboratorio iníciales destacaron alteraciones de función hepática, bilirrubina de $6,8 \mathrm{mg} \%$ (directa de 5,3 $\mathrm{mg} \%$ ).

Al evaluar a la madre se observó fenotipo sugestivo de hipertiroidismo, con delgadez marcada y exoftalmo, por lo que se sospechó enfermedad tiroidea no tratada (figura 2). Debido a sospecha de hipertiroidismo neonatal,

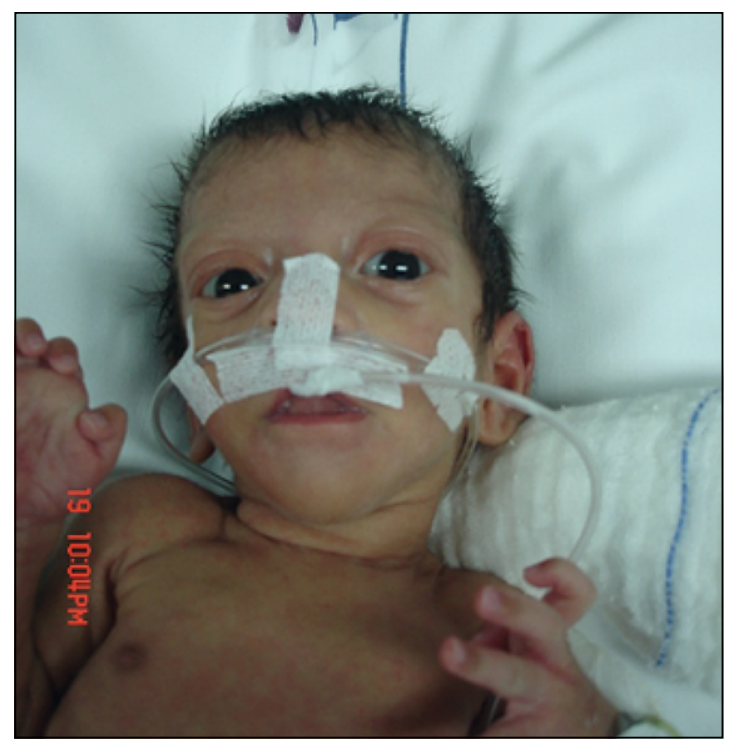

Figura 1. RN con restricción severa del crecimiento. Se nota el exoftalmo marcado y temblores recurrentes, con fenotipo similar a los hallazgos maternos durante el embarazo. (fotografía autorizada). se realizó perfil tiroideo, que mostró TSH 0,01 $\mathrm{uUI} / \mathrm{ml}$ y T4 Libre de 7,7 (valores de referencia $0,71-1,85 \mathrm{ng} / \mathrm{dl})$. Se realizaron además anticuerpos antimicrosomales, los cuales fueron negativos, pero no se pudo realizar anticuerpos estimulantes del receptor de tirotropina (TRA$\mathrm{ab})$.

Al reevaluar al paciente se observó bocio difuso que se confirmó con ecografía (figura 3). Se inició metimazol $1 \mathrm{mg} / \mathrm{kg} /$ día dividido en 3 dosis, propanolol a dosis de $1 \mathrm{mg} / \mathrm{kg} /$ día dividido en 2 dosis, logrando mejoría de

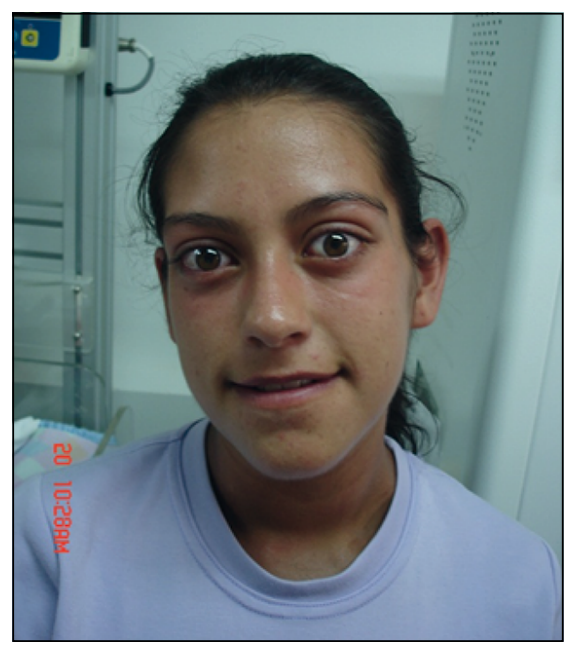

Figura 2. Madre con fenotipo sugestivo de hipertiroidismo. Nótese el exoftalmos, asociado a los síntomas referidos durante de la gestación (poca ganancia de peso, sudoración, frialdad distal entre otros), confirman la patología de base. (fotografía autorizada).

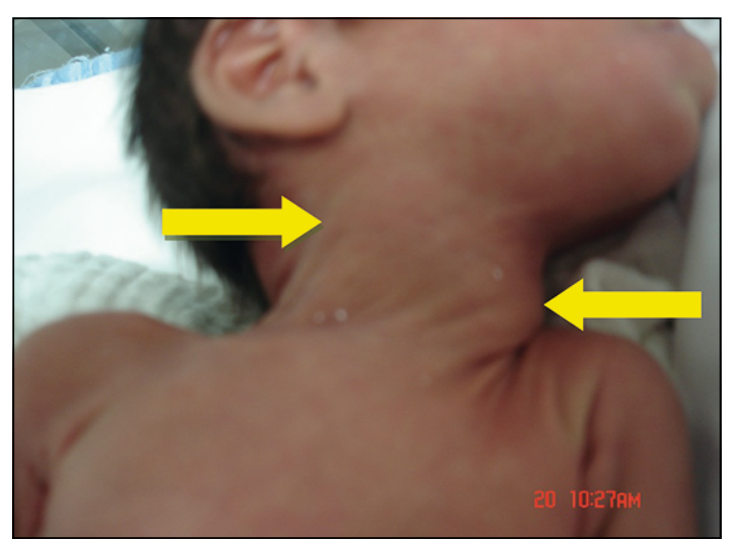

Figura 3. RN con aumento simétrico de la glándula tiroides. no hay signos inflamatorios ni obstrucción clínica de la vía aérea. 
la taquicardia. Sin embargo, se notó poca ganancia de peso en los primeros días de manejo, situación revertida después de una semana de tratamiento. Se logró retiro de oxígeno, resolución de hepatomegalia y después de 1 semana de tratamiento la T4 Libre descendió hasta 3,1 $\mathrm{mg} / \mathrm{dl}$, persistiendo con supresión de la TSH $(0,015 \mathrm{uUI} / \mathrm{ml})$ al momento de su egreso.

En la semana 13 de evolución se normalizaron las pruebas de función tiroidea, retirándose la medicación antitiroidea. Durante el seguimiento, el lactante permaneció en condición eutiroidea, sin alteraciones cardiovasculares ni del neurodesarrollo. La madre fue referida a endocrinología para manejo y asesoría para futuros embarazos.

\section{Discusión}

Fetos y neonatos de madres con enfermedad de Graves pueden presentar compromiso de su función tiroidea, tanto hipo como hipertiroidismo. Esto va a depender del predominio de anticuerpos inhibidores o estimuladores frente al receptor de tirotropina y de la terapia antitiroidea, la cual va a tener paso transplacentario ${ }^{8}$.

El hipertiroidismo fetal ocurre usualmente en el tercer trimestre de gestación, y la presentación neonatal es una rara condición, usualmente autolimitada y generalmente puede resolverse antes del cuarto mes, por el catabolismo de los anticuerpos de transferencia materna. Los síntomas son de expresión variable, y es probable que algunos recién nacidos, hijos de madres con hipertiroidismo autoinmune presenten hipertiroidismos transitorios no detectados 9 .

Las consecuencias a corto y largo plazo van a depender de la actividad (niveles anticuerpos estimulantes del tiroides) y duración del compromiso fetal.

En relación a la presencia de hipertiroidismo fetal, su diagnóstico se sospecha por la identificación de taquicardia fetal, aceleración de la maduración ósea, que puede llevar a microcefalia, abombamiento frontal, fascies triangular; lo anterior asociado al antecedente materno de enfermedad tiroidea, elevación de anticuerpos estimulantes del tiroides y ha- llazgos ultrasonográficos como aumento de la vascularización del tiroides y bocio fetal ${ }^{10}$. En formas más graves, puede aparecer falla cardíaca e hidrops. En el caso reportado, existió un compromiso fetal severo, dado por el fenotipo descrito y la repercusión clínica.

Generalmente se asocia al paso transplacentario de anticuerpos estimulantes de tiroides (inmune), pero pueden existir formas no inmunes, por alteraciones genéticas del receptor, las cuales pueden ser autosómicas dominantes o de aparición esporádica ${ }^{11}$. Lo anterior tendría repercusión clínica, ya que estas alteraciones genéticas, hacen del cuadro clínico, una condición permanente. De ahí que nuestro reporte a pesar de no tener anticuerpos TRAab, se comportó clínicamente como una forma inmune.

El manejo debe ser preventivo, identificando las gestantes con hipertiroidismo secundario a enfermedad de graves activa o antecedentes de enfermedad de Graves manejada con I131, tiroidectomia, condiciones no excepcionales y que están asociadas a riesgos materno-fetales y neonatales. El tratamiento del hipertiroidismo durante la gestación, se puede realizar con antitiroideos o cirugía, siendo el manejo médico la mejor opción. La droga de elección debe ser el propiluracilo, por el riesgo de embriopatía del metimazol. Hoy se sabe que los antitiroideos en general no están libre de efectos adversos, ya que pueden atravesar la placenta y desencadenar hipotiroidismo fetal ${ }^{12}$. Asociado a lo anterior, existen reportes de casos de hepatitis neonatal secundaria a tratamiento con propiuracilo $^{13}$.

Los hijos de madres con hipertiroidismo, sin ninguna intervención, tienen un riesgo hasta de 14 veces mayor de incidencia de malformaciones congénitas y complicaciones tempranas, por lo que la intervención interdisciplinaria debe ser prioritaria por todos los efectos devastadores mencionados ${ }^{14}$; de ahí las manifestaciones clínicas tan severas de nuestro reporte, asociado al alto riesgo neurológico en estos casos.

Por otro lado, se debe realizar cuantificación de anticuerpos estimulantes de tiroides, lo que determinaría el riesgo potencial de hipertiroidismo neonatal. Niveles de anticuerpos 3-5 veces sobre el valor normal, asociado a 
oftalmopatía materna, aumenta el riesgo para compromiso neonatal. Cabe destacar que los títulos de anticuerpos no se disminuyen con el tratamiento materno, por lo cual, se debe realizar la cuantificación de anticuerpos a madres con enfermedad de Graves activa, antecedentes de tratamiento con I131, tiroidectomía e hijos previos con hipertiroidismo neonatal ${ }^{15}$.

Los recién nacidos deben ser monitorizados clínicamente y con pruebas tiroideas durante las 2 primeras semanas de vida, ya que suelen ser asintomáticos los primeros días ${ }^{16}$. La identificación precoz y su manejo está relacionada con la prevención de complicaciones cardiovasculares y secuelas a largo plazo.

El cuadro clínico neonatal se manifiesta por síntomas adrenérgicos, como los comentados en nuestro paciente, con riesgos de miocardiopatía, colestasis y retinopatía. En el diagnóstico diferencial se debe considerar cardiopatía congénita, taquiarritmia, sepsis, síndrome de abstinencia, alteraciones neurológicas, entre otras $^{17}$.

Dado que el compromiso cardiovascular determina el pronóstico temprano ${ }^{18}$, la evaluación debe incluir ecocardiograma; este examen ayuda al diagnóstico diferencial con cardiopatía congénita al permitir una evaluación completa de la función cardíaca. El aumento de los niveles de hormona tiroidea está asociado con un incremento del volumen sanguíneo, contractilidad y frecuencia cardíaca, y una disminución de la resistencia vascular sistémica, por lo que se genera un estado hiperdinámico circulatorio y sobrecarga cardíaca ${ }^{19}$.

Adicional a la falla cardíaca, hay reportes de casos que evolucionan a hipertensión pulmonar severa, requiriendo terapias complejas de rescate como óxido nítrico inhalado, inotrópicos, hasta llegar a la circulación extracorpórea $(\mathrm{ECMO})^{20,21}$. Otro hallazgo relacionado es el compromiso isquémico potencial, asociado a vasoespasmo coronario e incremento de las demandas, en un miocardio expuesto a altos niveles de hormonas tiroideas. Este compromiso isquémico ha sido reportado en adultos, con un carácter reversible ${ }^{22}$. Por lo anterior, estudios electrocardiográficos y marcadores enzimáticos de isquemia deben hacer parte del estudio de los pacientes con diagnóstico de hi- pertiroidismo asociado a compromiso cardiovascular $^{23}$.

Una vez establecido el diagnóstico de hipertiroidismo neonatal, se debe iniciar el tratamiento antitiroideo, preferiblemente metimazol o carbimazol. Otra alternativa es el propiluracilo que además inhibe la conversión periférica de T4 a T3, sin embargo, hay reporte de hepatoxicidad asociado a su uso. Adicionalmente se puede asociar un beta bloqueante para controlar la hiperactividad muscular y cardíaca. Las alternativas pueden ser el propanolol, pero en caso de tormenta tiroidea y compromiso cardiovascular severo, la infusión endovenosa de esmolol puede ser una opción. El Iodo (lugol) y compuestos iodados pueden ser una alternativa, ante falta de control con los antitiroideos y beta bloqueadores. Por último en niños con poca respuesta a la terapia mencionada, los esteroides pueden tener algún beneficio, porque inhiben la conversión de T4 a T3 ${ }^{24}$. Otras alternativas planteadas son el uso de inmunoglobulina, por analogía de su uso en enfermedad inmune neonatal, como en la enfermedad hemolítica o púrpura trombocitopénico inmune ${ }^{25}$.

Se debe monitorizar en forma seriada el T3 y T4, y en base a esta, disminuir la medicación hasta suspenderla, siempre buscando evitar el hipotiroidismo iatrogénico, que puede alterar el desarrollo y maduración normal del recién nacido. La TSH es un mal marcador de seguimiento, ya que esta puede permanecer suprimida después de la resolución del cuadro clínico, como sucedió en nuestro paciente en etapas iniciales del manejo.

\section{Conclusiones}

Reportamos un recién nacido con falla cardíaca secundaria a hipertiroidismo inmune, hijo de una madre sin diagnóstico previo. El adecuado manejo de las enfermedades tiroideas durante el embarazo disminuye los riesgos de morbilidades en el período neonatal. Adicional a sus implicaciones tempranas, existen complicaciones neurológicas tardías, lo que hace indispensable que su manejo y seguimiento sea interdisciplinario, para disminuir la discapacidad. 


\section{Referencias}

1.- Price Jack F: R.E. Shaddy (ed.): Heart Failure in Congenital Heart Disease, 21DOI: 10.1007/978-1.

2.- Sharma M, Nair MNG, Jatana SK, Shahi BN: Congestive Heart Failure in Infants and Children. MJAFI 2003; 59: 228-33.

3.- Markham LA, Stevens DL: A case report of neonatal thyrotoxicosis due to maternal autoimmmune hyperthyroidism. Adv Neonatal Care 2003; 3 (6): 272-82.

4.- Hernández MI, Lee $K W$ : Neonatal Graves Disease Caused by Transplacental Antibodies Neo Reviews 2008; 9 : e305-9.

5.- Watkins MG, Dejkhamron P, Huo J, Vázquez DM, Menon RK: Persistent neonatal thyrotoxicosis in a neonate secondary to a rare thyroid-stimulating hormone receptor activating mutation: case report and literature review. Endocr Pract 2008; 14 (4): 479-83.

6.- Patil-Sisodia K, Mestman JH: Graves hyperthyroidism and pregnancy: a clinical update. Endocr Pract 2010; 16 (1): 118-29.

7.- Polak M, Le Gac I, Vuillard E, et al: Fetal and neonatal thyroid function in relation to maternal Graves' disease. Best Pract Res Clin Endocrinol Metab 2004; 18 (2): 289-302.

8.- García García E, Porras Cantarero JA, Fernández López P, Dominguez Gutiérrez de Ceballos R, Domínguez Pascual I: Hipertiroidismo neonatal en hijos de madres con enfermedad de Graves tratadas con radioyodo. Endocrinol Nutr 2013. Mar 11 http://dx.doi.org/10.1016/j. endonu.2012.11.007

9.- Papendieck P, Chiesa A, Prieto L, Gruñeiro-Papendieck $L$ : Thyroid disorders of neonates born to mothers with Graves' disease. J Pediatr Endocrinol Metab 2009; 22 (6): 547-53.

10.- Huel C, Guibourdenche J, Vuillard E, et al: Use of ultrasound to distinguish between fetal hyperthyroidism and hypothyroidism on discovery of a goiter. Ultrasound Obstet Gynecol 2009; 33 (4): 412-20.

11.- de Roux N, Polak M, Couet J, et al: A neomutation of the thyroid-stimulating hormone receptor in a severe neonatal hyperthyroidism. J Clin Endocrinol Metab 1996; 81 (6): 2023-6.

12.- Chattaway JM, Klepser TB: Propylthiouracil versus methimazole in treatment of Graves' disease during pregnancy. Ann Pharmacother 2007; 41 (6): 1018-22.

13.- Rivkees SA, Szarfman A: Dissimilar hepatotoxicity profiles of propylthiouracil and methimazole in children. $\mathrm{J}$
Clin Endocrinol Metab 2010; 95 (7): 3260-7.

14.- Gerrits $P$ : Heart failure in a neonate: commentary. Clin Pediatr (Phila) 2005; 44 (7): 638-9.

15.- Stagnaro-Green A, Abalovich M, Alexander E, et al: American Thyroid Association Taskforce on Thyroid Disease During Pregnancy and Postpartum. Guidelines of the American Thyroid Association for the diagnosis and management of thyroid disease during pregnancy and postpartum. Thyroid 2011; 21 (10): 1081-125.

16.- Polak M: Hyperthyroidism in early infancy: pathogenesis, clinical features and diagnosis with a focus on neonatal hyperthyroidism. Thyroid 1998; 8 (12): 1171-7.

17.- Männistö T, Mendola P, Reddy U, Laughon SK: Neonatal outcomes and birth weight in pregnancies complicated by maternal thyroid disease. Am J Epidemiol 2013; 178 (5): 731-40.

18.- Szalóczi B, Harmath A, Pete B, Kovács E, Rigó $J J$, Hajdú $J$ : Severe circulatory insufficiency in a patient with neonatal hyperthyroidism]. Orv Hetil 2010; 151 (12): 509-12.

19.- Lozano HF, Sharma CN: Reversible pulmonary hypertension, tricuspid regurgitation and right-sided heart failure associated with hyperthyroidism: case report and review of the literature. Cardiol Rev 2004; 12 (6): 299-305.

20.- Obeid R, Kumar Kalra V, Arora P, et al: Neonatal thyrotoxicosis presenting as persistent pulmonary hypertension. BMJ Case Rep 2012; 30; 2012.

21.- Oden J, Cheifetz IM: Neonatal thyrotoxicosis and persistent pulmonary hypertension necessitating extracorporeal life support. Pediatrics 2005; 115 (1): 105-8.

22.- Masani ND, Northridge DB, Hall RJ: Severe coronary vasospasm associated with hyperthyroidism causing myocardial infarction. Br Heart J 1995; 74 (6): 700-1.

23.- Trapali C, Dellagrammaticas HD, Nika A, Iacovidou $N$ : A unique case of reversible myocardial ischemia in a hyperthyroid neonate. Pediatr Cardiol 2008; 29 (1): 180-2.

24.- Bahn Chair RS, Burch HB, Cooper DS, et al: American Thyroid Association; American Association of Clinical Endocrinologists. Hyperthyroidism and other causes of thyrotoxicosis: management guidelines of the American Thyroid Association and American Association of Clinical Endocrinologists. Thyroid 2011; 21: 593-646.

25.- Peter F, Kalmar A, Kucsera R, et al: Human immunoglobulin therapy in serious neonatal Graves' disease. Long term (9 yrs) follow up [abstract]. Horm Res 2005; 64 (Suppl 1): 249-50. 